$>$ Les anomalies génétiques à l'origine des maladies hémorragiques ont permis de découvrir la plupart des protéines de l'hémostase. Par analogie avec l'hémophilie, on a utilisé le terme de thrombophilie pour désigner un excès de coagulation responsable de thrombose. Les premiers cas de thrombophilie héréditaire décrits dans la littérature sont les déficits en antithrombine, protéine $C$ et protéine $S$, trois inhibiteurs de la coagulation. Il s'agit d'anomalies à transmission autosomique dominante se manifestant chez l'adulte par des thromboses veineuses profondes récidivantes. Ces déficits sont rares, alors que les thromboses veineuses ne le sont pas. Avec la découverte de deux mutations fréquentes, le FV Leiden et la mutation g.20210 G $\rightarrow$ A du gène de la prothrombine (F2), apparaît la notion de facteur de prédisposition génétique dont l'impact est démontré par des études épidémiologiques, mais dont la traduction clinique en termes de thrombose est beaucoup plus complexe. <

\title{
Mutations des protéines de la coagulation et thromboses
}

Martine Aiach, Martine Alhenc-Gelas, Delphine Borgel, Joseph Emmerich, Sophie Gandrille, Véronique Picard

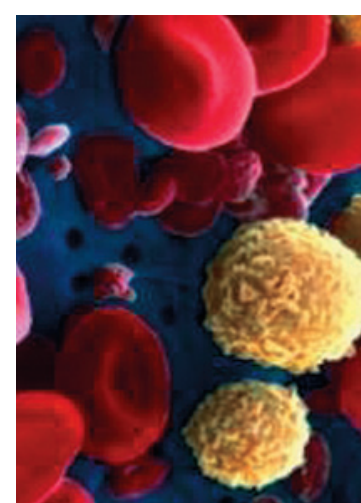

tes inactives - les facteurs (F) de la coagulation II, VII, IX, X et XI qui développent une activité catalytique de type sérineprotéase en présence de deux cofacteurs (FV et FVIII). Le déclenchement se fait grâce au facteur tissulaire (FT), une protéine membranaire présente sur la plupart des cellules mais dont l'expression est réprimée dans les cellules du sang circulant et les cellules endothéliales vasculaires. Toute blessure vasculaire entraîne l'exposition du FT et la fixation du FVII activé (a) présent en petite quantité dans le sang circulant. Les complexes FT-FVIla activent d'autres molécules de FVII et les FX et IX. Les premières traces de thrombine formée par l'action du FXa sur la prothrombine (FII) vont permettre la mise en place de plusieurs boucles d'amplification: (1) l'activation des plaquettes qui exposent des phospholipides anioniques procoagulants; (2) I'activation du FV qui se fixe sur les phospholipides anioniques en même temps que le FXa, engendrant un complexe enzymatique - la prothrombinase - capable d'activer beaucoup plus efficacement la prothrombine; (3) l'activation du FVIII qui s'assemble avec le FIXa et le FX sur les phospholipides anioniques pour accélérer la production du FXa; (4) l'activation du FXI pour renforcer l'activation du FIX. Ce système d'interactions complexes permet la pro-

d'activation de la coagulation contribuent fréquemment à la constitution d'une thrombose veineuse.

La coagulation met en jeu une série de protéines circulan-

Article reçu le 13 mars 2006, accepté le 30 juin 2006. 
duction d'une concentration élevée de thrombine capable de transformer le fibrinogène soluble en un réseau de fibrine polymérisée. L'intervention d'une transglutaminase, le FXIII activé par la thrombine, crée des liaisons de covalence entre les monomères de fibrine et stabilise le thrombus formé de cellules sanguines prisonnières d'un réseau de fibrine insoluble.

Le système de la coagulation, normalement destiné à colmater les blessures vasculaires, est contrôlé par plusieurs mécanismes inhibiteurs (Figure 1). L'antithrombine (AT) est une serpine (serine-protease inhibitor), qui forme avec la thrombine, le FXa et le FIXa des complexes inactifs de stœchiométrie $1: 1$. La vitesse de formation de ces complexes est fortement augmentée par l'héparine et les sulfates d'héparane des cellules endothéliales. La protéine $C(P C)$ est activée par la thrombine fixée par la thrombomoduline, une protéine exprimée à la membrane des cellules endothéliales. L'activation de la PC est favorisée par sa fixation à l'EPCR (endothelial cell protein C receptor). $\varepsilon n$ présence d'un cofacteur, la protéine S (PS), la PC activée ( $P C a$ ) clive plusieurs ponts peptidiques du FVa et du FVIIla. Ainsi, le complexe $P C a-P S$ ralentit-il la production de thrombine par inactivation de ces deux facteurs.

Un équilibre subtil maintient normalement la fluidité du sang circulant. Les concentrations circulantes des facteurs participant à la coagulation et à sa régulation présentent des variations inter-individuelles,

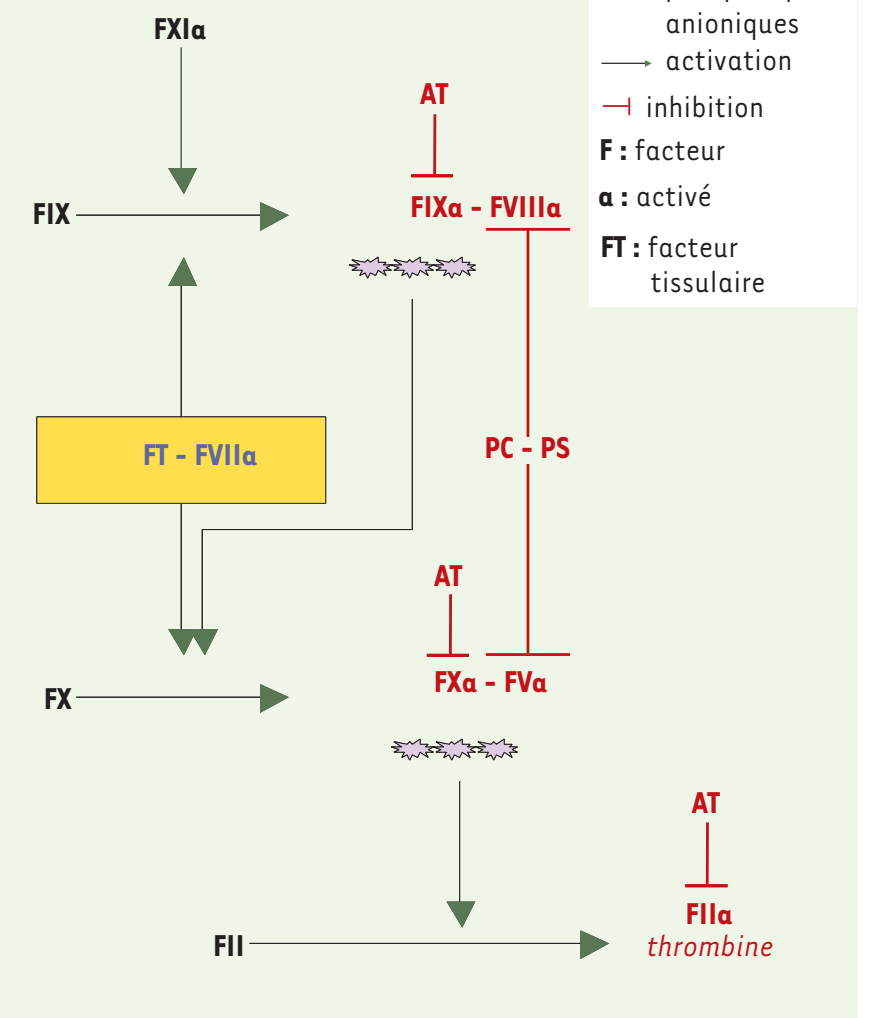

Figure 1. Mécanisme de la régulation de la production de thrombine par l'antithrombine (AT), la protéine $C(P C)$ et la protéine S (PS).
I'hérédité contribuant de façon non négligeable à leur variance, comme cela a été observé par l'analyse de cohortes de jumelles homozygotes et l'étude de grandes familles [1-3]. Il existe donc un contexte génétique dont l'impact est, pour l'instant, difficile à définir chez un individu donné.

\section{La thrombophilie héréditaire, une maladie rare due à un déficit en inhibiteurs de la coagulation}

Les thromboses veineuses profondes (TVP) affectent le plus souvent les membres inférieurs, avec un risque de migration du thrombus qui conduit à l'embolie pulmonaire, les deux aspects étant regroupés dans le terme de maladie thrombo-embolique veineuse (MTEV). L'existence de MTEV familiales a été décrite depuis longtemps et la recherche d'anomalies de la coagulation dans ces familles dites thrombophiliques a permis d'identifier, en 1965 [4], le premier cas de déficit héréditaire en antithrombine : une diminution de $50 \%$ de l'aptitude à neutraliser la thrombine est observée chez dix membres d'une famille dont six avaient souffert d'une MTEV, suggérant une transmission autosomique dominante et une pénétrance clinique incomplète. La MTEV peut survenir chez l'adulte jeune (le risque est de l'ordre de $1 \%$ par an chez les hétérozygotes) en l'absence d'autres facteurs de risque. La prévalence est de l'ordre de 0,02\% dans la population générale.

Le gène de l'AT, AT3, est localisé sur le chromosome 1 et s'étend sur 1,3 kb. L'analyse des fondements moléculaires du déficit montre qu'il s'agit le plus souvent de mutations privées et ponctuelles réparties sur les sept exons, entraînant une perte de fonction [5]. Un groupe de mutations faux-sens affectant les exons 2 et 3 codant pour le site de fixation de l'héparine est associé à un phénotype biologique particulier dit type II HBS (heparin binding site) : la protéine a une aptitude normale à neutraliser la thrombine et les autres sérineprotéases de la coagulation, mais elle ne fixe pas l'héparine. Les sujets hétérozygotes pour ce type de mutation ne présentent pas de prédisposition à la thrombose veineuse. En revanche, une quinzaine d'homozygotes ont été décrits dans la littérature avec un phénotype clinique sévère associant des thromboses veineuses et artérielles qui peuvent survenir dès l'enfance [6].

Un petit nombre de mutations affectent la structure de la protéine qui a tendance à se polymériser. Ce phénomène, qui inactive la protéine, pourrait entraîner un effet dominant négatif chez les hétérozygotes, notamment lors d'épisodes fébriles. Nous avons eu l'occasion d'identifier, chez un enfant de 13 mois ayant présenté une thrombose veineuse cérébrale à l'issue d'un épisode 
infectieux, une mutation entraînant clairement la polymérisation in vivo. Cet enfant est homozygote [7], ses parents sont consanguins et asymptomatiques.

Les déficits en protéine $C$ décrits quelques années plus tard [8] sont plus fréquents que le déficit en AT ( $0,2 \%$ à $0,4 \%$ dans la population générale). La pénétrance avec une incidence de MTદV de l'ordre de 0,5\% par patientannée est relativement faible: $50 \%$ des patients sont asymptomatiques à l'âge de 45 ans. Dans cette forme classique du déficit en PC, la transmission est autosomique dominante. II existe toutefois une forme à transmission récessive beaucoup plus sévère et précoce [8] dont l'incidence se situe entre $1 / 500000$ et $1 / 750000$ naissances. Les enfants homozygotes présentent, dans les jours qui suivent la naissance, des thromboses capillaires entraînant des nécroses cutanées étendues évoquant un purpura fulminans, associées à des thromboses veineuses et parfois même artérielles.

L'analyse du gène de la $P C, P R O C$, qui s'étend sur $11 \mathrm{~kb}$ et comprend 9 exons, a permis d'identifier de nombreuses mutations, la plupart du temps ponctuelles et privées [9]. II n'existe pas de mutations associées à des formes cliniques particulières, notamment à la transmission récessive. Beaucoup de mutations fauxsens n'annulent pas totalement l'expression du gène et leur caractère délétère est difficile à prouver compte tenu de la pénétrance faible. II faut noter l'existence de familles avec des sujets hétérozygotes composites qui ont des concentrations diminuées ( $10 \%$ à $50 \%$ ) et une symptomatologie qui peut être relativement sévère, avec une MTEV précoce avant l'âge adulte alors que les hétérozygotes, porteurs de l'une ou l'autre mutation, sont asymptomatiques et présentent des concentrations subnormales de PC [10].

Le déficit en protéine $S$ se présente de la même façon que le déficit en $\mathrm{PC}[11]$. Il est difficile à reconnaître sur le plan biologique car $60 \%$ de la PS circule sous forme d'un complexe avec la chaîne $\beta$ de la $C 4 b$ binding protein et seuls les $40 \%$ libres sont actifs. Le mécanisme d'action de la PS reste assez mystérieux. C'est un cofacteur peu puissant de la PCa, tout au moins in vitro. Le déficit en PS libre entraîne néanmoins dans les familles étudiées une MTEV chez l'adulte, avec une pénétrance de $50 \%$ à l'âge de 45 ans. II existe quelques cas publiés de transmission récessive avec le même tableau dramatique dans la période néonatale que celui observé dans les déficits homozygotes en PC.

L'analyse du gène de la PS, PROS1, qui s'étend sur $80 \mathrm{~kb}$ et comprend 15 exons, est compliquée par la présence d'un pseudogène. De nombreuses mutations ont été identifiées, mais aucune relation claire entre le type de mutation et la pénétrance clinique n'a pu être démon- trée [12]. Les quelques cas (sept à ce jour dans la littérature) de présentation sévère néonatale avec des taux de PS indétectables sont homozygotes.

En pratique, le diagnostic de déficit en AT, PC et PS est fondé essentiellement sur un test fonctionnel évaluant l'activité biologique dans le plasma, principal phénotype intermédiaire. Le dosage immunologique permet de confirmer le diagnostic et de différencier les déficits quantitatifs (les plus fréquents) des déficits qualitatifs. L'analyse du gène est justifiée dans les cas d'homozygotie suspectée (activité biologique $<10 \%$ ) ou en cas de phénotype intermédiaire complexe et difficile à interpréter.

\section{Le FV Leiden et le F2 g.20210A : deux facteurs génétiques fréquents de thrombophilie}

À côté des mutations des gènes AT3, PROC et PROSI qui sont rares et hétérogènes, deux mutations entraînant un gain de fonction sont observées avec des fréquences de l'ordre de $5 \%$ pour le FV Leiden et $3 \%$ pour F2 g.20210A [13]. Ces deux anomalies génétiques sont dues à un effet fondateur et la mutation, survenue il y a 20000 à 30000 ans, affecte les populations caucasiennes. Elles ont été découvertes grâce à un phénotype intermédiaire qui en explique l'association avec la MTEV [14, 15]. Dans le cas du FV Leiden, la mutation transforme I'Arg506 en GIn, I'un des sites de clivage du FVa par la PCa, et entraîne une résistance à l'effet anticoagulant de la PCa in vitro $[14,16]$. Ce phénotype de résistance à la $P C$ a pu être utilisé pour le diagnostic biologique. La mutation F2 g.20210 G>A affecte la région 3' de l'ARN messager et augmente l'efficacité de la traduction du précurseur de la thrombine. On observe une légère augmentation de la concentration circulante de prothrombine, mais la variabilité inter-individuelle est trop importante pour que sa mesure soit un marqueur fiable de la mutation.

La prévalence de la mutation du gène du FV Leiden, identifiée en 1994, a permis de confirmer dans des études rétrospectives son association à un sur-risque de TVP. Le risque relatif est de l'ordre de 4,9 (IC 4,1$5,9)$ dans une méta-analyse de huit études cas-témoins totalisant 2310 patients et 3204 témoins [17]. Environ $20 \%$ des patients ayant souffert d'au moins un épisode de TVP sont porteurs d'un FV Leiden. Ce risque est vérifié dans des études prospectives de cohortes [18] et de membres asymptomatiques de familles thrombophiliques porteurs de la mutation [19]. Les homozygotes (1 pour 2500 sujets dans la population générale) ont un risque relatif plus élevé (de l'ordre de 20) mais présentent la même symptomatologie que les hétérozygotes, avec un début à l'âge adulte et une pénétrance qui reste incomplète [20]. Plusieurs études confirment une observation qui peut sembler paradoxale: l'embolie pulmonaire est deux fois moins fréquente chez les sujets symptomatiques porteurs d'un FV Leiden [21]. Une explication possible est la formation d'un thrombus plus solide et plus adhérent diminuant le risque de migration.

La mutation F2 g.20210 G>A, découverte en 1996, a également permis des études épidémiologiques nombreuses qui montrent un risque relatif de 3,8 (3,0-4,9) dans la méta-analyse citée précédemment. Environ 
$10 \%$ des patients avec MTEV sont porteurs de cette mutation. L'homozygotie est rare et ne semble pas être associée à un risque beaucoup plus élevé.

II n'existe pas de justification au diagnostic présymptomatique du FV Leiden ou de F2 g.20210A. La question se pose après un premier épisode de TVP. Les études épidémiologiques portant sur le risque de récidive chez les porteurs de l'une de ces deux mutations après un premier épisode de TVP ne montrent pas d'effet de ces mutations à l'état hétérozygote sur le risque de récidive, qui est important chez tous les patients, quel que soit leur statut génétique.

\section{La thrombophilie: une maladie oligogénique et multifactorielle}

L'analyse de nombreuses familles thrombophiliques porteuses d'anomalies des gènes $A T 3, P R O C$ et PROS1 a permis de montrer que $10 \%$ à $30 \%$ des sujets symptomatiques étaient également porteurs du FV Leiden [22-25]. De même, l'association du FV Leiden avec F2 g.20210A a un effet synergique dans la méta-analyse des études cas-témoins [17]. Les sujets porteurs de plusieurs allèles à risque sont ainsi, dans l'ensemble, les plus fréquemment symptomatiques. Cette complexité explique pourquoi l'histoire familiale n'est pas toujours informative quand on s'intéresse aux phénotypes cliniques. La perspective de découverte de nouveaux facteurs génétiques de risque de thrombose s'est révélée relativement décevante au cours des dix dernières années, pratiquement tous les gènes candidats ayant été criblés sans succès dans des familles thrombophiliques, avec ou sans anomalies identifiées. Une augmentation du FVIII circulant est clairement associée à la MTદV dans plusieurs études, sans que les fondements génétiques de cette augmentation n'aient été, pour l'instant, établis. Des polymorphismes modifiant la fonctionnalité du FXIII [26] ou de l'EPCR [27] sont faiblement associés à la MTEV dans certaines études, mais d'autres études ne retrouvent pas cette association.

L'utilisation de méthodes modernes de criblage du génome n'a pas, pour l'instant, permis de progrès décisif.

\section{Conclusions}

La MTEV est une maladie fréquente chez l'adulte ( 1 cas pour 1000 par an). Les facteurs circonstanciels favorisant sa survenue sont nombreux: l'âge, la grossesse, l'immobilisation prolongée, la chirurgie (en particulier orthopédique). Les traitements hormonaux (contraceptifs oraux, traitement substitutif de la ménopause) sont également impliqués. L'exposition à ces facteurs de risque exogènes est un facteur déclenchant chez près de la moitié des sujets symptomatiques porteurs d'une anomalie génétique. Ainsi, dans les études portant sur le risque de MTEV chez des femmes utilisant des contraceptifs oraux, on observe un risque beaucoup plus élevé chez les femmes porteuses d'un FV Leiden, suggérant un effet synergique [28]. On connaît les effets des œstrogènes sur les protéines de la coagulation ; la modification de l'équilibre de la coagulation par un facteur exogène serait ainsi moins bien tolérée chez les sujets porteurs d'un facteur de risque génétique. Cette hypothèse est renforcée par des observations récentes. $\varepsilon$ n effet, une augmentation des marqueurs d'activation de la coagulation s'observe principalement chez les femmes porteuses de la mutation Leiden du FV et/ou en association avec la prise d'une contraception œstroprogestative [29]. Ainsi, l'interaction de cinq facteurs de risque génétiques bien identifiés avec des facteurs de risque acquis, et éventuellement d'autres facteurs génétiques restant à découvrir, détermine l'apparition du phénotype thrombophilique. $\diamond$

\section{SUMMARY}

Coagulation factor mutations and thrombosis

The coagulation system is governed by a subtle balance between clotting activators and inhibitors. Many genes can contribute to the overall phenotype, and polymorphisms may act to up regulate or down regulate the generation of thrombin, the coagulation-key enzyme. An increase in coagulation factor (gain function) or/and a decrease in coagulation inhibitors (loss of function) may favor venous thromboembolism (VTE). It has been observed since a long time that VTE may be a familial disease, but it was only in 1965 that Egeberg published the first case of inherited antithrombin (AT) deficiency. This was followed by similar reports of protein C (PC) and protein S (PS) deficiencies. Hereditary thrombophilia was thus initially considered as a rare monogenic disorder with incomplete penetrance. AT, PC and PS deficiencies are due to multiple and mostly private mutations of the corresponding genes. Most patients are heterozygous and experience VTE at adult age. Homozygosity associated with severe thrombosis at birth has been observed in newborns with undetectable PC or PS concentrations. The discovery of factor (F) V Leiden and F2 g.20210 G>A, two gain of function mutations, challenged the view of thrombophilia as a rare monogenic disorder. FV Leiden and F2 g.20210 G>A are due to a founder effect and affect populations of European descent with frequencies at $5 \%$ and $3 \%$ respectively. These two mutations are moderate of risk factor for thrombosis and paved the way for gene-gene and gene-environment interactions. Patients carrying more than one genetic risk factor are at higher risk to develop VTE. The exposition to acquired risk factors such as estrogene based oral contraception may also have a synergistic effect favoring thrombosis in patients with FV Leiden or other genetic risk factors. $\diamond$

\section{RÉFÉRENCES}

1. De Lange M, Snieder H, Ariens RA, et al. The genetics of haemostasis: a twin study. Lancet $2001 ; 357: 101-5$.

2. Ariens RA, de Lange M, Snieder $H$, et al. Activation markers of coagulation and fibrinolysis in twins : heritability of the prethrombotic state. Lancet $2002 ; 359: 667-71$.

3. Souto JC, Almasy L, Borrell M, et al. Genetic determinants of hemostasis phenotypes in Spanish families. Circulation 2000 ; 101 : 1546-51. 
4. Egeberg 0 . Inherited antithrombin deficiency causing thrombophilia. Thromb Diath Haemorr $1965 ; 13: 516-30$.

5. Lane DA, Bayston T, Olds RJ, et al. Antithrombin mutation database: $2^{\text {nd }}$ (1997) update. For the plasma coagulation inhibitors subcommittee of the scientific and standardization committee of the International society on thrombosis and haemostasis. Thromb Haemost 1997 ; 77 : 197-211.

6. Kuhle S, Lane DA, Jochmanns K, et al. Homozygous antithrombin deficiency type II (99 Leu to Phe mutation) and childhood thromboembolism. Thromb Haemost 2001 ; 86 : 1007-11.

7. Picard V, Dautzenberg MD, Villoutreix B0, et al. Antithrombin Phe229Leu a new homozygous variant leading to spontaneous antithrombin polymerization in vivo associated with severe childhood thrombosis. Blood 2003; 102 : 919-25.

8. Griffin JH, Evatt B, Zimmerman TS, et al. Deficiency of protein C in congenital thrombotic disease. J Clin Invest 1981; 68 : 1370-3.

9. Reitsma PH, Bernardi F, Doig RG, et al. Protein C deficiency: a database of mutations, 1995 update. On behalf of the subcommittee on plasma coagulation inhibitors of the scientific and standardization committee of the ISTH. Thromb Haemost $1995 ; 73: 876-89$.

10. Gandrille S, Aiach M. Identification of mutations in 90 of 121 consecutive symptomatic French patients with a type I protein C deficiency. The French Inserm network on molecular abnormalities responsible for protein C and protein S deficiencies. Blood $1995 ; 86: 2598-605$.

11. Comp PC, Nixon RR, Cooper MR, Esmon CT. Familial protein $S$ deficiency is associated with recurrent thrombosis. J Clin Invest 1984 ; 74 : 2082-8.

12. Gandrille $S$, Borgel $D$, Ireland $H$, et al. Protein $S$ deficiency : a database of mutations. For the plasma coagulation inhibitors subcommittee of the scientific and standardization committee of the International society on thrombosis and haemostasis. Thromb Haemost 1997 ; 77 : 1201-14.

13. Seligsohn U, Lubetsky A. Genetic susceptibility to venous thrombosis. $N$ Engl J Med 2001 ; $344: 1222-31$.

14. Dahlback B, Carlsson M, Svensson PJ. Familial thrombophilia due to a previously unrecognized mechanism characterized by poor anticoagulant response to activated protein $C$ : prediction of a cofactor to activated protein C. Proc Natl Acad Sci USA 1993 ; 90 : 1004-8.

15. Poort SR, Rosendaal FR, Reitsma PH, Bertina RM. A common genetic variation in the 3'-untranslated region of the prothrombin gene is associated with elevated plasma prothrombin levels and an increase in venous thrombosis. Blood $1996 ; 88: 3698-703$.

16. Bernardi F, Faioni $\varepsilon M$, Castoldi $\varepsilon$, et al. A factor $V$ genetic component differing from factor $V$ R $506 \mathrm{Q}$ contributes to the activated protein $C$ resistance phenotype. Blood $1997 ; 90: 1552-7$
17. Emmerich J, Rosendaal FR, Cattaneo M, et al. Combined effect of factor V Leiden and prothrombin 20210A on the risk of venous thromboembolism--pooled analysis of 8 casecontrol studies including 2310 cases and 3204 controls. Study group for pooled-analysis in venous thromboembolism. Thromb Haemost 2001; 86: 809-16.

18. Ridker PM, Hennekens $\mathrm{CH}$, Lindpaintner $\mathrm{K}$, et al. Mutation in the gene coding for coagulation factor $\mathrm{V}$ and the risk of myocardial infarction, stroke, and venous thrombosis in apparently healthy men. $N$ Engl J Med 1995 ; $332: 912-7$.

19. Martinelli I, Mannucci PM, De Stefano V, et al. Different risks of thrombosis in four coagulation defects associated with inherited thrombophilia: a study of 150 families. Blood $1998 ; 92: 2353-8$.

20. Rosendaal FR, Koster T, Vandenbroucke JP, Reitsma PH. High risk of thrombosis in patients homozygous for factor V Leiden (activated protein C resistance). Blood $1995 ; 85$ : 1504-8.

21. Bounameaux $H$. Factor $V$ Leiden paradox : risk of deep-vein thrombosis but not of pulmonary embolism. Lancet $2000 ; 356: 182-3$.

22. Koeleman BP, Reitsma PH, Allaart CF, Bertina RM. Activated protein C resistance as an additional risk factor for thrombosis in protein C-deficient families. Blood 1994 ; 84 : 1031-5.

23. Gandrille S, Greengard JS, Alhenc-Gelas M, et al. Incidence of activated protein C resistance caused by the ARG 506 GLN mutation in factor V in 113 unrelated symptomatic protein Cdeficient patients. The French network on the behalf of Inserm. Blood $1995 ; 86: 219-24$

24. Van Boven HH, Vandenbroucke JP, Briet $\varepsilon$, Rosendaal FR. Gene-gene and gene-environment interactions determine risk of thrombosis in families with inherited antithrombin deficiency. Blood 1999; $94: 2590-4$.

25. Borgel D, Duchemin J, Alhenc-Gelas M, et al. Molecular basis for protein S hereditary deficiency : genetic defects observed in 118 patients with type I and type Ila deficiencies. The French network on molecular abnormalities responsible for protein $\mathrm{C}$ and protein $\mathrm{S}$ deficiencies. J Lab Clin Med 1996; 128 : 218-27.

26. Alhenc-Gelas M, Reny JL, Aubry ML, et al. The FXIII Val 34 Leu mutation and the risk of venous thrombosis. Thromb Haemost $2000 ; 84: 1117-8$.

27. Saposnik B, Reny J, Gaussem P, et al. A haplotype of the EPCR gene is associated with increased plasma levels of sEPCR and is a candidate risk factor for thrombosis. Blood 2004 ; $103: 1311-8$

28. Bloemenkamp KW, Rosendaal FR, Helmerhorst FM, Vandenbroucke JP. Higher risk of venous thrombosis during early use of oral contraceptives in women with inherited clotting defects. Arch Intern Med $2000 ; 160: 49-52$.

29. Schreijer AJM, Cannegieter SC, Meijers JCM, et al. Activation of coagulation system during air travel : a crossover study. Lancet $2006 ; 367: 832-8$

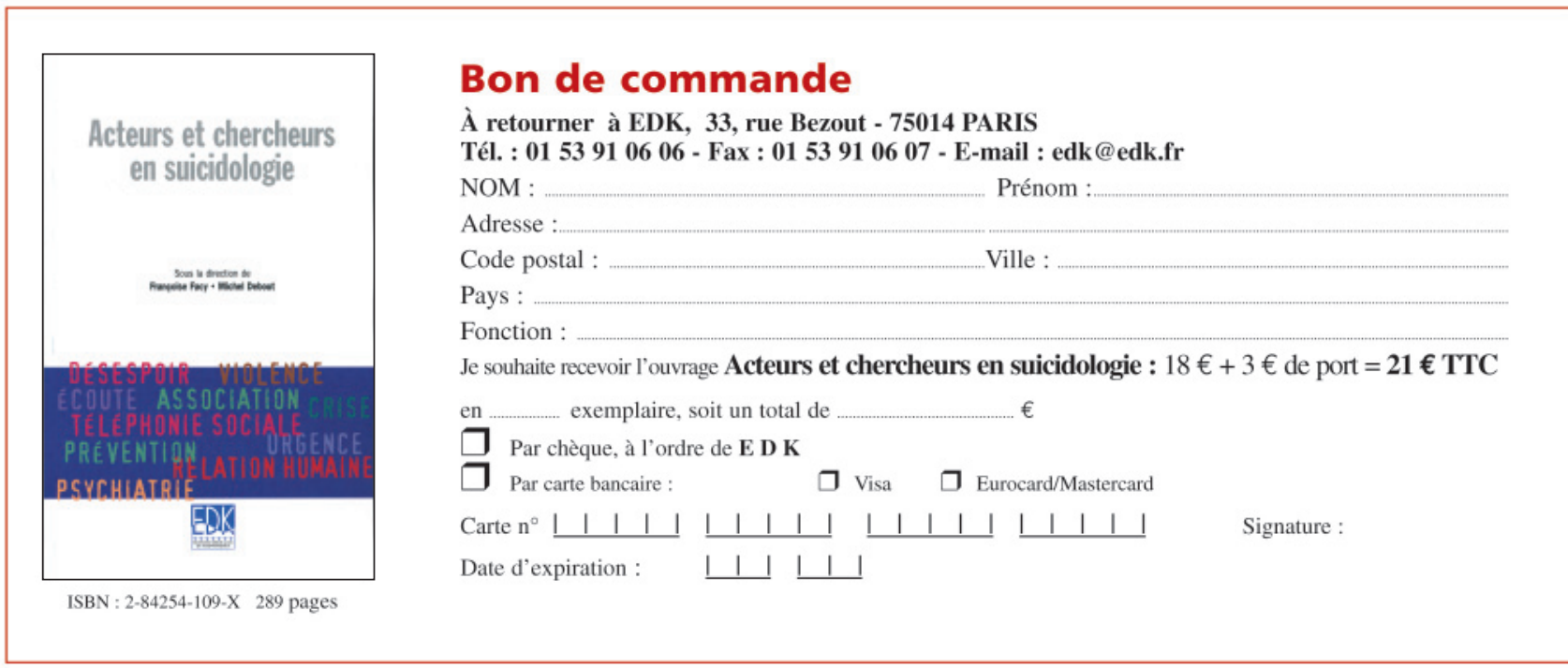

